\title{
Automated business process management - in times of digital transformation using machine learning or artificial intelligence
}

\author{
Daniel Paschek ${ }^{1, *}$, Caius Tudor Luminosu ${ }^{1}$, and Anca Draghici ${ }^{1}$ \\ ${ }^{1}$ Politehnica University Timisoara, Management Faculty, 300191, Timisoara, Romania
}

\begin{abstract}
The continuous optimization of business processes is still a challenge for companies. In times of digital transformation, faster changing internal and external framework conditions and new customer expectations for fastest delivery and best quality of goods and many more, companies should set up their internal process at the best way. But what to do if framework conditions changed unexpectedly? The purpose of the paper is to analyse how the digital transformation will impact the Business Process Management (BPM) while using methods like machine learning or artificial intelligence. Therefore, the core components will be explained, compared and set up in relation. To identify application areas interviews and analysis will be held up with digital companies. The finding of the paper will be recommendation for action in the field of BPM and process optimization through machine learning and artificial intelligence. The Approach of optimizing and management processes via machine learning and artificial intelligence will support companies to decide which tool will be the best for automated BPM.
\end{abstract}

\section{Introduction}

The ongoing industrial revolution from the implementation of mechanic production over the division of labour and assembly line work to the usage of electronic and automatic production were the first three named types of industrial revolution [1]. Actually we arrived at the 4.0 step of the industrial revolution - the so-called digitalization [2]. Behind the concepts Smart Factory, Internet of Things, intelligent manufacturing, Cloud Computing, Big Data, Machine to Machine communication and many more, new elements and trends affect the established economy [3]. Mc Kinsey advises therefore: "The traditional manufacturing business model is changing, and new models are emerging; incumbents must be quick to recognize and react to these new competitive challenges" [3].

Driven by the Internet, real and virtual worlds are growing ever further to form an internet of things and cloud computing to import [4]. Cloud computing is hosted on the internet and allows remote access to apps, services, and stored to manage processes, workflows or machines [5]. The internet of things is described by J. Brixel as: "The internet of things builds on this concept by using the cloud to store and automate processes in

\footnotetext{
*Corresponding author: paschi88@gmx.net
} 
objects that are synced to the internet, like internet-enabled automobiles and remote home lighting and shade systems" [5]. In a nutshell, Industry 4.0 takes processes and the management of processes which are normally managed internally by people into the cloud where they can be adapted and analysed from anywhere [5].

In times of Big Data, constantly changing conditions in the supply chain and new customer requirements, process management and automation play a major role to reduce response time, to avoid back logs, dissatisfied clients and processing errors [6].

To enable a further step in process management and automation this research looks in detail at the possibilities of using machine learning or artificial intelligence for Business Process Management (BPM) in times of digital transformation. The opportunity of independent process management, via automated and learning computer programs promises in theory agility, flexibility and automation for a whole company as well as the process stake holders. The aim of this paper is to investigate the application these two mentioned developments in the field of Business Process Management. The outcome will show the effect as well as advantages and disadvantages of machine learning and artificial intelligence for process steering and optimization.

\section{Scientific status of the research}

\subsection{Definition of Digital Transformation}

The term digital transformation exists since the 1960s, with the wider availability of mainframe computers [7]. Over further development steps in digitalization like in 1980 the e-mail or 1990 the emergence of CRM tools and Business Intelligence (BI) to connected objects, 3D printing, the block chain technology and artificial intelligence (AI), the innovation in digital technologies and digital transformation changed the way of collaboration, execution at the level of the whole economy in a radical way [7].

In a nutshell, digital transformation means high-speed changes in the cooperation due to innovation and implementation of digital technologies and the integration into all aspects of human life and society at different dimensions [8].

\subsection{Definition of Business Process Management}

Business Process Management (BPM) is a management concept for controlling, adapting and optimizing business processes [9]. A concrete definition for BPM from the European Association of Business Process Management (EABPM) defined BPM as a systematic approach, to capture, shape, execute, document, measure, monitor and steering automatic and non-automatic processes to reach coordinated and sustainable company targets [10]. The aim of Business Process Management (BPM) is to improve the corporate performance by optimizing and managing business process of the company [11].

Business Process Management can be described as corporate business process optimization and management over single systems like ERP, CRM and SCM and the whole integrated network.

The assignment of a BPM-System is furthermore the coordination and execution of business processes step by step [11]. Through monitoring, identifying and evaluating of process issues or troubles, the BPM-System depicts where a process had trouble and enables the business to optimize their process issue [11]. 


\subsection{Definition of Artificial Intelligence}

In the literature are two different approaches to define the artificial intelligence (AI). On one hand humans are drawn as comparison and on the other AI is characterized by the list of different application and work areas [12]. For example:

- "the theory and development of computer systems able to perform tasks normally requiring human intelligence..." [12];

- "The AI examines how to capture and understand the intelligent behaviour of computers, or how to solve problems by using computers that require interoperability "[12];

- "AI is a subdivision of computer science, which deals with the investigation of different problem areas like robotic, speech and flow text recognition as well as image and video processing [12].

In the scope of this paper AI can be defined as a section of informatics and applied computer science to pattern human proceedings of problem solving and transfer them to computers in order to invent efficient and new solutions as well as course of actions. Therefore, $\mathrm{AI}$ is a computer program running on any possible device(s) or data centre(s) with the skill to interact with its environment.

The definition of AI suffers from the fact that the terms "intelligence" and "intelligent human behaviour" are not well defined and understood yet [13]. In 1950 the British mathematician A.M. Turing (1912-1954) wrote the critical essay "Computing Machinery and Intelligence" on the subject of AI. He asks the question of whether a program can be intelligent and invented the so-called Turing test [13]. In this experiment, a test person communicates via a computer terminal with two non-visible partners, a program and a human being [13]. Finishing, the test person must decide which is which.

Two types of AI are distinguished in the literature like described in the following figure. It's important to understand the differentiation of AI for the following paper.

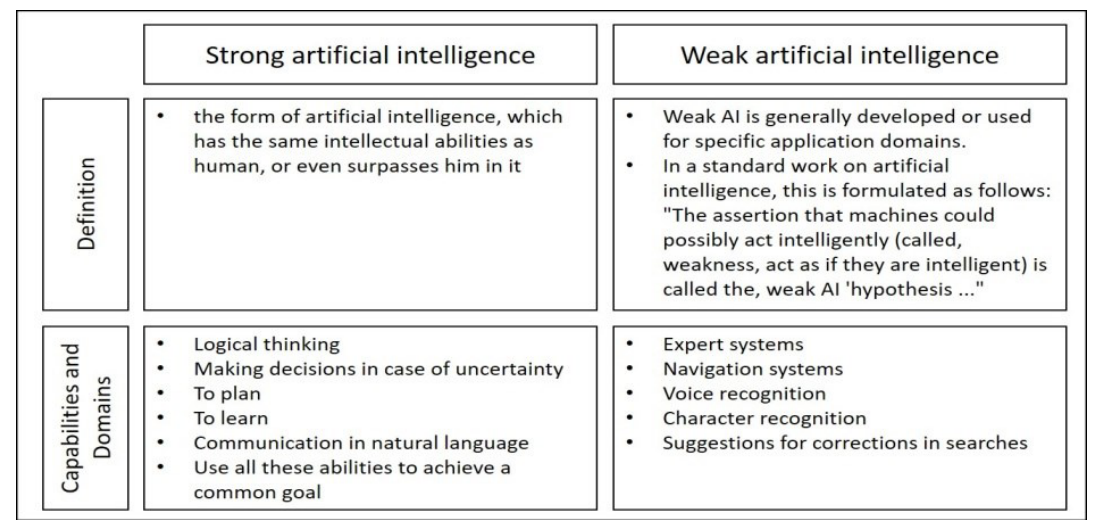

Fig. 1. Strong vs. Weak AI, own presentation based on [14]

Already today AI can be found in different types of weak AI in the human environment. Examples of this are object-oriented programming languages and graphical user interfaces, translation systems as well as "intelligent games" like chess can now be installed on almost all computers. Chess programs have recently defeated the best human players, like the IBM Deep Blue Super Computer [15]. Further samples of AI are voice recognitions and control systems like Cortana, Siri or Echo or intelligent bots and Agents who interact independently in phone conversations. The recent successes in AI are enabled by a very broad field of machine learning [15]. 


\subsection{Definition of Machine Learning}

Machine learning is a branch of AI and describes mathematical techniques that enable a system, that is, a machine, to independently generate knowledge from experience. [16] Machine Learning describes the development of algorithms based on empirical data as well as training data with the focus on the optimization of the results and the improvement of the predictions due to learning processes [16]. The term algorithm can be defined as detailed sequences of actions to solve a particular task [15]. The development of machine learning is due to the study of recognition patterns and computer learning theory [15]. The algorithmic implementation of machine learning takes place with monitored or unsupervised learning like shown in the following figure 2 .

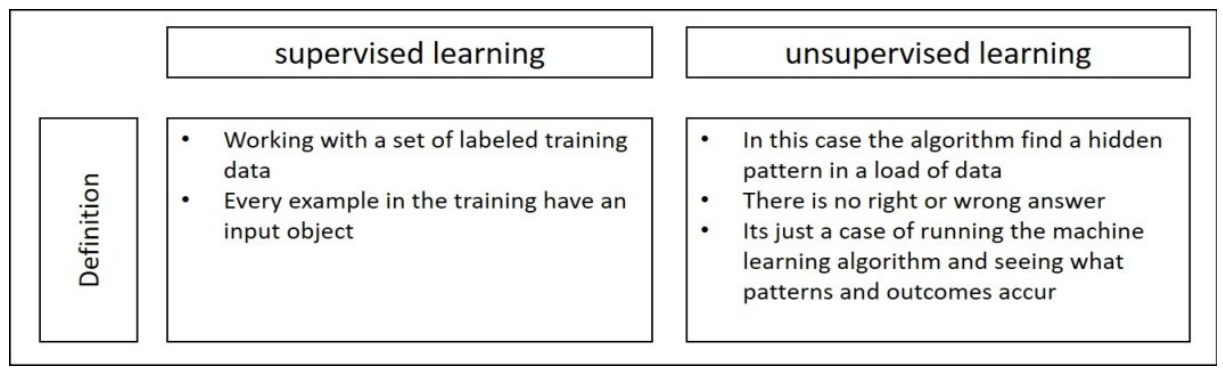

Fig. 2. Supervised and unsupervised Learning, own presentation based on [16]

In the past, the automatic processing of written and spoken language was attempted with the most comprehensive set of rules without any significant progress. [15] Nowadays, the methods of "deep learning" are used in areas of Natural Language Processing (NLP), above all for language recognition.

In part, the terms "deep learning" and artificial neural networks are used synonymously [15]. Deep learning works with artificial neural networks in order to achieve particularly efficient learning sequences like the language recognition "Siri" [15] The process eliminates the need for many neural network operations because the computer takes all intermediate steps. [15] The Scientist has only to present data to the neural network, such as images. How these are to be identified, the net finds completely by itself [15]. The neural networks are divided into several layers, like a filter that works from the coarse to the fine, thus increasing the likelihood of detection and output of a correct result [15]. Deep learning methods first learn low-level elements such as brightness values, then mid-level elements, and finally high-level elements like whole faces, [15]. These incremental enhancement is illustrated at the following figure 3 .

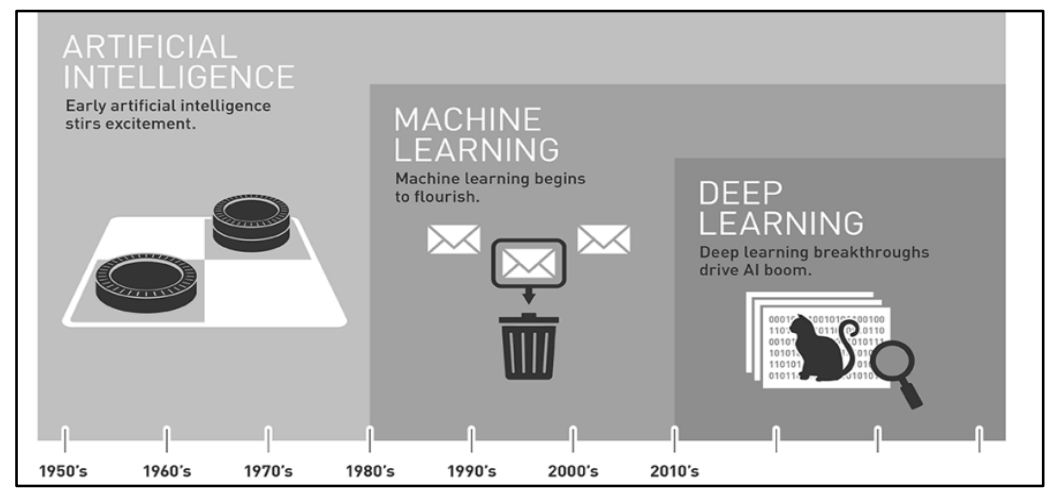

Fig. 3. Incremental enhancement, source: [17] 


\subsection{Evaluation of scientific status}

The theory shows that methods of "deep learning" with neural networks are always meant when artificial intelligence is mentioned. The rapid increased progress of "deep learning" over the past years can mainly lead back to more powerful hardware for computing operations [15]. Furthermore the rising amounts of data which are important for the initial training of the neural Networks have positive effects [17]. After initial training, the "deep learning" of the procedure consists in always learning during the running application, which increases the recognition accuracy and results. From this can be deducted that next to the algorithm the big data plays a significant role for the deep learning and thus for the artificial intelligence.

\section{The Research Methodology}

For the proposed evaluation and analysis of using machine learning or AI a survey with interview characteristics and a shadowing with so-called digital companies was executed to identify the status quo and execution status at the economy to this topic. Based on the analysed facts and the AI approach a recommendation was announced to the survey participants and their companies for automated process optimization with machine learning or AI.

\subsection{Detailed study description}

For the analysis of the base data a field study and survey was carried out at 25 digital companies in Germany. A digital company is characterized by the preponderant use of information technology for their primary business activities within their value-added step. More than $75 \%$ of the survey questions were focused on the business process execution and the application of information technologies. Part of the survey was the possibility for an assessment of the daily work by shadowing the Process Management Team. Shadowing means to attend the participant at his daily business to have a look the work. For the study the anonymous survey method was used.

It was ensured that the subjects present their knowledge, experiences, expectations and there actually execution of process management. Target persons were mainly Chief Information Officers (CIO), Chief Technology Officers (CTO), Chief Execution Officer (CEO), Chief Digital Officer (CDO) and IT process managers. The base line is set up by 65 valid survey replies from a total of 75 requested ones. The survey embraced 30 closes and 5 open questions. The survey via interview proceedings took approximately 35 till 45 minutes per person.

Next to the survey a shadowing was requested at the selected companies. As observer were the process flows, the process management tasks, the processes itself as well as the necessary applications watched and analysed. The time duration of the shadowing differs via company between 4 and 8 hours.

Afterwards a comprehensive analyses and comparison of the collected data take place to identify the base line as well as deviations. Through the shadowing the analysis of the theoretical appreciation and the execution was possible.

\subsection{Research and Interview results}

In the following, the results of the survey are presented and the recommendations for using machine learning or AI are mentioned. $87 \%$ of the requested Managers of 25 companies 
allowed the survey and interview as well as the shadowing to analyse the base line of execute automated BPM.

The survey shows that every company and $100 \%$ of the respondents are affected by the digitalization. $59 \%$ of these are concerned very strong, $35 \%$ strong and only $6 \%$ stated low. These results indicate that the digital transformation and information technology plays a major role in the companies. This can be underpinned by the results which show in what state of digital transformation the survey participants are. Because approximately $38 \%$ are at the growth phase, $51 \%$ at the transition phase and only $11 \%$ at the beginning of the implementation of the digitalization.

Further relevant research results are listed in the following.

- $39 \%$ of the respondents worked in the IT branch, $19 \%$ at professional services, $18 \%$ at the retail, $15 \%$ at Logistics as well as $9 \%$ at the bank sector

- The selected companies can classify in 52\% family business and middle class business, $27 \%$ Start-up and Internet business and $21 \%$ enterprise business

- $51 \%$ of the respondent's rate data of the company, like process data as strategic assets and the foundation of the optimization. 39\% assess data as feasible data and privacy problem and only $9 \%$ are sceptical and see data as cost drivers

- $100 \%$ of the attendees know and uses BPM in the company and $85 \%$ want more process optimizations and automated BPM applications

- only $12 \%$ uses data warehouses, business intelligence systems or data lakes for data reporting and data management

- $75 \%$ of the respondents know the terms $\mathrm{AI}$ and machine learning but only $29 \%$ assess machine learning actually

- just $26 \%$ have already heart about deep learning and just $2 \%$ execute it

Very interesting are the survey results concerning the utilisation of machine learning now and in the future and AI like shown in the following figure 4.

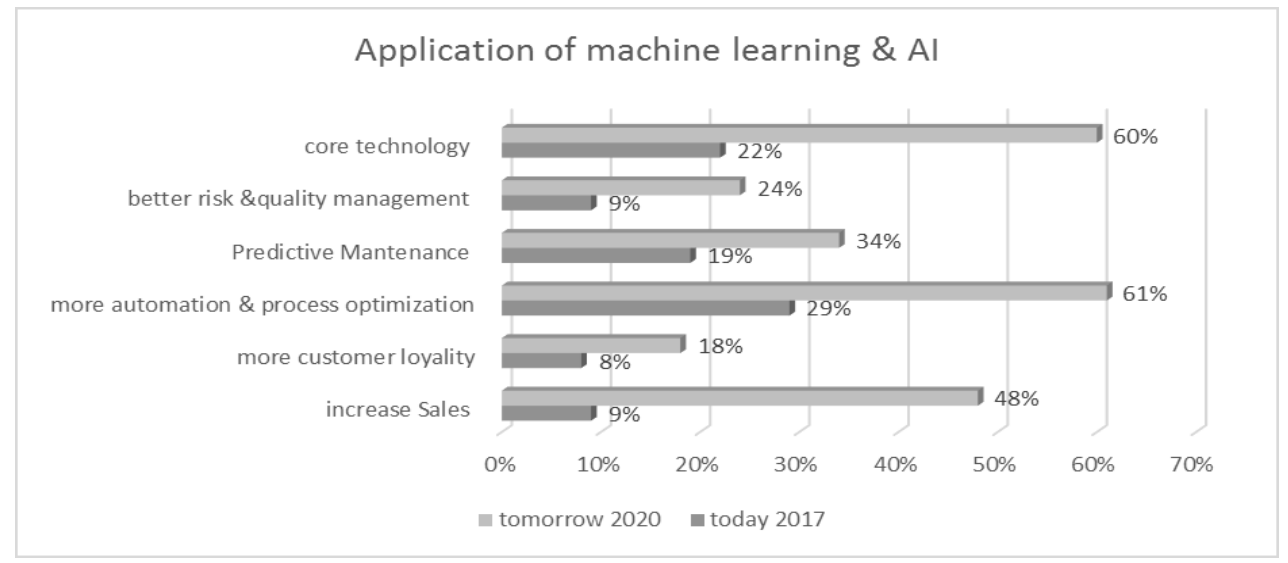

Fig. 4. Application of machine learning \& AI, own presentation

It can be concluded that process optimization with $29 \%$ is the most important and assessed application for the participants. Other applications like machine learning as core technology for new business models or predictive maintenance are the second and third important areas of application. The outlook and estimation to the year 2020 shows the strengthened position of the desire to more process optimization and more automation. Also, the increased value of the application in core technologies shows the meaning of the continuing digitalisation. The biggest step in the field of machine learning can be conducted at the sales. 
The findings of the shadowing are very different between the own assessment of the participants. As benchmark, the elevated data from the survey and interview will be set. As it can be seen in the following figure 5, most participants evaluate their use of BPM as up to date and their process automation and controlling as well set up.

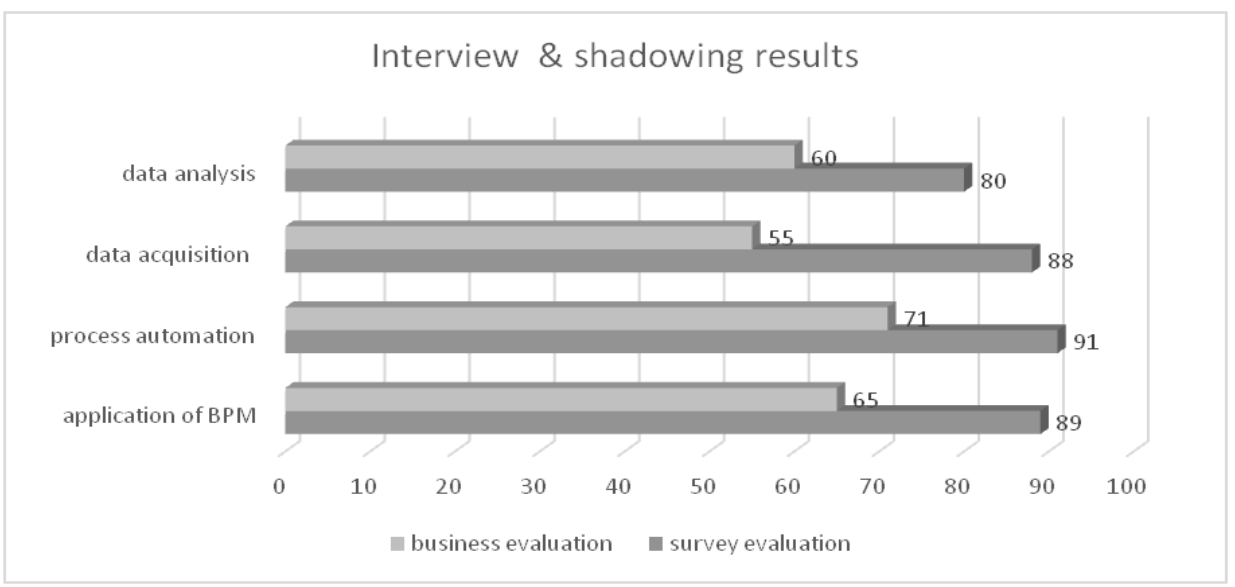

Fig. 5. Interview vs. shadowing results, own presentation

While the survey assessment generates $87 \%$ of all questions in figure five. The compared value while the shadowing is $15 \%$ lower by $62 \%$. This shows the different exceptions and the big value for improvement. Particularly the process automation point shows a difference of $20 \%$ in theory and business. Such gaps can result in big problems in cases of missed BPM and missing continual improvement.

To avoid business faults, half automatic processes and the waste of resources, the base data regarding a special field like processes have to be set anew by the companies. In connection with detailed data analysis, deep learning and machine learning the optimization and automation opportunities can be pointed out.

\subsection{Pre-Conclusion}

For $80 \%$ of the participants deep learning, machine learning and AI are synonyms for the same concept. Like described in the theoretical part, machine learning is regarded as a subarea of artificial intelligence.

The results of the survey show the demand for machine learning and AI for the optimization and automation of the processes and the support of BPM. Furthermore, the participants evaluate the application of machine learning as very important in the future. Not only for process optimization, but rather as core competency for new business models and for an increase in sales support.

The results of the shadowing show two main findings:

1. the participants estimate their use of BPM and automated process management better than it is in reality

2. approximately $45 \%$ of the compiled data from the companies are not used or controlled effectively

This shows a big opportunity and space for improvements only in the evolution of process management. Big Data and data lakes are used in the business but the reporting and data analysis can be enhanced. Deep learning, machine learning and AI can be named as a recommendation to automate and optimize the process and they support the BPM through new algorithms and independent learning via constant data analysis. 


\section{Conclusion}

Digitization is unstoppable and changes the market situation in many business sectors. Time-intensive manual workflows, based on e-mail, Excel or well and truly paper work reduce the productivity of companies. August Wilhelm Scheer, Managing Director of the Scheer Group stated already in 2015: "Everything that can be digitized will be digitized. Each process, product and business model becomes digital, so the revolution lies in the processes." [18] This quote underlines the opportunity for an optimized process management through digitalization and the execution of automated processes supported by AI. Therefore, targeted and consistent process automation is a critical success factor now and in the future as it can be seen by the evaluation of the survey results. It is becoming increasingly important for companies to use the power of their data and to make smart and profitbale decisions.

\section{References}

1. K. Schwab, Die Vierte Industrielle Revolution, (Publisher Pantheon Verlag, 2016)

2. Ö. Atiker, In einem Jahr digital: Das Praxishandbuch für die digitale Transformation, (Publisher Viley-VCH Verlag \& Co.KGaA 2017)

3. http://www.mckinsey.com/business-functions/operations/our-insights/manufacturings-next-act Accesed 2015

4. http://www.hightech-strategie.de/de/Industrie-4-0-59.php Accesed 2017

5. https://www.salesforce.com/uk/blog/2016/09/what-is-industry-4-0.html Accesed 2016

6. http://ipt.ch/digitalisierung-mit-prozessautomatisierung-beginnen/ Accesed 2016

7. J.L. Leignel,T. Ungaro, and A. Staar, Digital Transformation: Information System Governance, (Publisher Wiley 2016)

8. D. Schallmo, A. Rusnjak, J. Anzengruber, and T. Werani, M. Jünger, Digitale Transformation von Geschäftsmodellen: Grundalgen, Instrumente und Best Practices, (Publisher Springer Gabler, 2016)

9. J.v. Brocke, and M. Rosemann, Handbook on Business Process Management 2, (Publisher Springer, 2015)

10. European Association of Business Process Management - EABPM, Business Process Management, Common Body of Knowledge, Gießen, (2009)

11. J.H. Schmelzer, W. Sesselmann, Geschäftsprozessmanagement in der Praxis.8th Edition, (Publisher Carl Hanser Verlag, 2013)

12. U. Lämmel, and J. Cleve, Künstliche Intelligenz, (Publisher Hanser, 2012)

13. R. Urwin, Artificial Intelligence - The quest of the ultimate thinking machine, (Publisher Arcturus Holdings Limited 2016)

14. http://www.informatik.unioldenburg.de/ iug08/ki/Grundlagen Starke KI vs. Schwache KI.ht $\underline{\mathrm{ml}}$ Accesed 2017

15. $\mathrm{http} / / /$ de.dice.com/nachrichten/aktueller-stand-kuenstliche-intelligenz/ Accesed 2016

16. J. Bell, Machine Learning: Hands-On for Developers and Technical Professionals, John Wiley \& Sons Inc., (2014)

17. https://blogs.nvidia.com/blog/2016/07/29/whats-difference-artificial-intelligence-machinelearning-deep-learning-ai/ Accesed 2016

18. http://www.me-netzwerk.de/wp-content/uploads/2015/05/Industrie-4.0-mit-Scheer-BPaaS- ME-Forum-Berlin.pdf Accesed 2015 